Terr. Atmos. Ocean. Sci., Vol. 18, No. 4, 777-803, October 2007

\title{
Conservative Semi-Lagrangian Scheme Applied to One-Dimensional Shallow Water Equations
}

\author{
Wen-Yih Sun ${ }^{1,2, *}$ \\ (Manuscript received 26 May 2006, in final form 14 February 2007)
}

\begin{abstract}
A forward-in-time semi-Lagrangian scheme developed by Sun et al. (1996) and Sun and Yeh (1997) has been applied to one-dimensional shallow water equations in both rotational and irrotational systems. After obtaining numerical results, we employ variation formulations (Sun and Sun 2004) with minimum correction to adjust both total mass and total energy so that they are conserved. Therefore, the scheme produces accurate, positive-definite solutions while conserving both mass and total energy. Comparing among different resolutions, the improvement on total energy is significant but less significant for a mass field in a coarse resolution model when it simulates the sharp discontinuities of surface waves, because the mass field calculation is quite accurate even without correction. The variation method proposed here can also be easily applied to multi-dimensional flows.
\end{abstract}

(Key words: Shallow water equations, Geostrophic adjust, Surface waves, Semi-Lagrangian scheme)

\section{INTRODUCTION}

Since 1959, semi-Lagrangian schemes have been studied by Wiin-Nielsen (1959) and many other scientists. Most semi-Lagrangian schemes employing backward trajectories have been reviewed by Staniforth and Côté (1991). Iterations are often required to solve the departure

\footnotetext{
1 Department of Earth and Atmospheric Sciences, Purdue University, West Lafayette, USA

2 National Center for High Performance Computing and Preparatory National Center for Typhoon and Flooding Research, Hsinchu, Taiwan, ROC

* Corresponding author address: Prof. Wen-Yih Sun, National Center for High Performance Computing and Preparatory National Center for Typhoon and Flooding Research, Hsinchu, Taiwan, ROC;

E-mail:wysun@purdue.edu doi: 10.3319/TAO.2007.18.4.777(A)
} 
points of the backward trajectories if the velocity field is not constant, as discussed by Bates (1984), Kuo and Williams (1990), Bermejo and Staniforth (1992), and Huang (1994). On the other hand, forward trajectories can easily be obtained with great accuracy (Purser and Leslie 1994). Thus, Purser and Leslie (1991) have introduced the 'cascade interpolation method'. Sun et al. (1996) have presented the 'split interpolation method with clipping filter', and Sun and Yeh (1997) have developed a more general 'internet interpolation method' with unconditional stability, which has also been successfully applied to the NTU-Purdue nonhydrostatic atmospheric model (Hsu and Sun 2001; Sun and Hsu 2005) by Shieh et al. (2006)

The use of semi-Lagrangian (SL) advection methods allows for relatively long time steps. It gives minimal phase error, minimizes the computational dispersion, and can handle sharp discontinuities (Nair and Machenhauer 2002). However, semi-Lagrangian does not guarantee the conservation of mass (Rancic 1992; Priestley 1993), which is important for a numerical model to simulate passive-scalar transport in the atmosphere or the properties of climate studies and ocean circulation (Priestley 1993). Hence, Rancic (1992), Priestley (1993), Gravel and Staniforth (1994), Lin and Rood (1996), Bermejo and Conde (2002), and others have developed different mass conserving, semi-Lagrangian schemes. The detailed mass conserving, semi-Lagrangian scheme derived from the piecewise parabolic method (PPM) based on the finite-volume scheme is referred to Rancic (1992). Priestley (1993) and Gravel and Staniforth (1994) applied a localized correction on the quasi-monotone semi-Lagrangian (QMSL) scheme of Bermejo and Staniforth (1992) to achieve mass conservation for a shallow water equation. On the other hand, Bermejo and Conde (2002) applied the variation formulation to achieve a global conservation of the QMSL. Based on Sun et al. (1996) and Sun and Yeh (1997), Sun and Sun (2004) (will be referred as SS hereafter) also applied the variation formulation with mass correction to obtain accurate, positive-definite, mass conserved solutions with simpler calculations. Furthermore, SS's results are as good as or more accurate than those obtained by Carpenter et al. (1990), Priestley (1993), and Bermejo and Conde's (2002) compared with analytical solutions for scalar, linear advection. Sun et al. (1996) have also proved that the accuracy of their semi-Lagrangian scheme is comparable or better than Kuo and Williams (1990) and others in solving nonlinear advection. Here, we apply the method developed by SS to one-dimensional shallow water equations. The simulations show that the scheme is capable of conserving both mass and total energy in rotational and irrotational systems. We are also applying this method to the two-dimensional shallow water equations to study interactions of vortices over topography based on the characteristic approach (Hirsch 1988, 1990; Wang and Yeh 2005) and the cases presented by Wang and Yeh (2005). They will be presented in other papers. It is also noted that Arakawa and Lamb (1981) and Thuburn and Staniforth (2004) have developed an elegant finite difference scheme to conserve mass, energy, and absolute potential vorticity in shallow water equations.

\section{SHALLOW WATER EQUATIONS AND NUMERICAL SCHEME}

\subsection{Basic Equations and Semi-Lagrangian Scheme}

The one-dimensional shallow water equations with the Coriolis force are: 


$$
\begin{aligned}
& \frac{\partial h}{\partial t}+u \frac{\partial h}{\partial x}+h \frac{\partial u}{\partial x}=0, \\
& \frac{\partial u}{\partial t}+u \frac{\partial u}{\partial x}+g \frac{\partial h}{\partial x}=f v, \\
& \frac{\partial v}{\partial t}+u \frac{\partial v}{\partial x}=-f u,
\end{aligned}
$$

where $h$ is the height. If we apply the time-split method, (2.1) - (2.3) can be solved as a combination of advection terms and pressure gradient forces [(2.4) - (2.6)], as well as the terms associated with the Coriolis force [(2.7) - (2.8)]. They are:

$$
\begin{aligned}
& \frac{\partial h}{\partial t}+u \frac{\partial h}{\partial x}+h \frac{\partial u}{\partial x}=0, \\
& \frac{\partial u}{\partial t}+u \frac{\partial u}{\partial x}+g \frac{\partial h}{\partial x}=0, \\
& \frac{\partial v}{\partial t}+u \frac{\partial v}{\partial x}=0,
\end{aligned}
$$

and

$$
\begin{aligned}
& \frac{\partial u}{\partial t}=f v, \\
& \frac{\partial v}{\partial t}=-f u .
\end{aligned}
$$

Following Stoker (1957) and Yih (1979) and introducing $c=(g h)^{1 / 2}$, we can replace (2.4) and (2.5) by

$$
\begin{aligned}
& \frac{\partial(u+2 c)}{\partial t}+(u+c) \frac{\partial(u+2 c)}{\partial x}=0, \\
& \frac{\partial(u-2 c)}{\partial t}+(u-c) \frac{\partial(u-2 c)}{\partial x}=0,
\end{aligned}
$$


or in a Lagrangian form,

$$
\begin{aligned}
& d(u+2 c) / d t=0, \text { along } d x / d t=u+c, \\
& d(u-2 c) / d t=0, \text { along } d x / d t=u-c,
\end{aligned}
$$

and from (2.6), we obtain:

$$
d v / d t=0 \text {, along } d x / d t=u \text {. }
$$

Inspection of (2.11) - (2.12) reveals that the quantity $u \pm 2 c$ is conserved along $d x / d t=u \pm c$, or they mean that "message" $u \pm 2 c$ is propagated through the fluid with speed $\pm c$ as well as carried with speed $u$ (Yih 1979). On the other hand, $v$ is conserved and carried by speed $u$ alone.

Let us define the solutions obtained from (2.11) - (2.12) as $p_{1}=u^{*}+2 c^{*}, p_{2}=u^{*}-2 c^{*}$, and from (2.13) as $v^{*}$, where $c^{*}=\left(g h^{*}\right)^{1 / 2}$. Then, $u^{*}$ and $h^{*}$ (before including the effect of rotation) can be determined as follows (Yih 1979; Erbes 1993):

$$
\begin{aligned}
& u^{*}=\frac{p_{1}+p_{2}}{2}, \\
& h^{*}=\left(\frac{p_{1}-p_{2}}{4 g^{1 / 2}}\right)^{2} .
\end{aligned}
$$

Then, apply $u^{*}$ and $v^{*}$ in equations (2.7) - (2.8), we obtain the analytic solution:

$$
\hat{u}=u * \cos (f \Delta t)+v * \sin (f \Delta t),
$$

and

$$
\hat{v}=v * \cos (f \Delta t)-u * \sin (f \Delta t)
$$

According to Sun et al. (1996) and Sun and Yeh (1997), the procedure to solve (2.11) - (2.13) includes:

(a) Constructing the Lagrangian network induced by the motion of the fluid from the Eulerian network and finding the intersections of the networks by a general interpolation from the irregularly distributed Lagrangian grid to the regularly distributed Eulerian grid;

(b) Applying the spatial filter to remove the unwanted short waves and the values beyond the constraints. 


\subsection{Conservation of Mass}

This section follows the procedures discussed in SS closely. Suppose the height at time $t_{n}$ is $h_{j}^{n}$, then the summation of $h_{j}^{n}$ over the entire domain of $j m$ grids is:

$$
H^{n}=\sum_{j m} h_{j}^{n} \delta x_{j}
$$

where $H^{n}$ is the total mass at $n^{\text {th }}$-time step, and $\delta x_{j}$ is the space interval at $j^{\text {th }}$ grid and can be a variable. The summation of $h^{*}$ in (2.15) is:

$$
H^{*}=\sum_{j m} h_{j}^{*} \delta x_{j}
$$

where the referenced values $h_{\max }$ and $h_{\min }$ are the extreme values of $h_{j}^{n}$ and can be functions of time and space also, as discussed in SS, we propose a mass correction function, $\delta h_{j}$, at the $j^{\text {th }}$ grid, given by the polynomial:

$$
\delta h_{j}=a\left(h_{\max }-h_{j}^{*}\right)\left(h_{\min }-h_{j}^{*}\right)+b\left(h_{\max }-h_{j}^{*}\right)\left(h_{\min }-h_{j}^{*}\right)\left(\bar{h}-h_{j}^{*}\right),
$$

where $\bar{h}=0.5\left(h_{\min }+h_{\max }\right)$, and $a$ and $b$ are determined by the following two equations:

$$
\sum_{j m} \delta h_{j} \delta x_{j}=\left[H^{n}+\Delta t\left(u_{1}^{n+1 / 2} h_{1}^{n+1 / 2}-u_{j m}^{n+1 / 2} h_{j m}^{n+1 / 2}\right)\right]-H^{*}=\delta H
$$

and

$$
\sum_{j m}\left(\delta h_{j}\right)^{2} \delta x_{j}=\mathfrak{I} \quad \text { (should be minimal) , }
$$

where $\Delta t\left(u_{1}^{n+1 / 2} h_{1}^{n+1 / 2}-u_{j m}^{n+1 / 2} h_{j m}^{n+1 / 2}\right)$ is the net mass flux between $t=n \delta t$ and $t=(n+1) \delta t$, which is ignored in this paper. Equation (2.21) guarantees the conservation of mass. Equation (2.22) implies a minimal modification of the results obtained by the original Semi-Lagrangian scheme. Equation (2.20) is one of the simplest equations with two undetermined parameters required to satisfy both (2.21) and (2.22). It is noted that (2.20) approaches zero when $h_{j}^{*}$ approaches $h_{\max }$ or $h_{\min }$ to ensure no overshooting of the results, which cannot be achieved by a lower-order polynomial. From (2.21) and (2.22), we can obtain:

$$
a=\frac{\delta H-b \Sigma_{3}}{\Sigma_{2}},
$$


and

$$
\begin{aligned}
b & =\sum_{j m}\left\{\left[\left(\frac{\delta H \Sigma_{3}}{\Sigma_{2}^{2}}\right)\left(h_{\text {max }}-h_{j}^{*}\right)^{2}\left(h_{\text {min }}-h_{j}^{*}\right)^{2}-\left(\frac{\delta H}{\Sigma_{2}}\right)\left(h_{\max }-h_{j}^{*}\right)^{2}\left(h_{\text {min }}-h_{j}^{*}\right)^{2}\left(\bar{h}-h_{j}^{*}\right)\right] \delta x_{j}\right\} \\
& \div \sum_{j m}\left\{\left[\left(\frac{\Sigma_{3}}{\Sigma_{2}}\right)^{2}\left(h_{\max }-h_{j}^{*}\right)^{2}\left(h_{\min }-h_{j}^{*}\right)^{2}+\left(h_{\max }-h_{j}^{*}\right)^{2}\left(h_{\min }-h_{j}^{*}\right)^{2}\left(\bar{h}-h_{j}^{*}\right)^{2}\right.\right. \\
& \left.\left.-\left(\frac{2 \Sigma_{3}}{\Sigma_{2}}\right)^{2}\left(h_{\max }-h_{j}^{*}\right)^{2}\left(h_{\min }-h_{j}^{*}\right)^{2}\left(\bar{h}-h_{j}^{*}\right)\right] \delta x_{j}\right\}
\end{aligned}
$$

where

$$
\Sigma_{2}=\sum_{j m}\left(h_{\max }-h_{j}^{*}\right)\left(h_{\min }-h_{j}^{*}\right) \delta x_{j},
$$

and

$$
\Sigma_{3}=\sum_{j m}\left(h_{\max }-h_{j}^{*}\right)\left(h_{\min }-h_{j}^{*}\right)\left(\bar{h}-h_{j}^{*}\right) \delta x_{j}
$$

Hence, after calculating $b$ from (2.24), and $a$ from (2.23), we obtain from $\delta h_{j}(2.20)$. Thus, the height of the $j$-th grid at time $t_{n+1}$ is:

$$
h_{j}^{n+1}=h_{j}^{*}+\delta h_{j},
$$

which guarantees that:

$$
H^{n+1}=\sum_{j m} h_{j}^{n+1} \delta x_{j}=\sum_{j m} h_{j}^{*} \delta x_{j}+\sum_{j m} \delta h_{j} \delta x_{j}=H^{*}+\delta H=H^{n}
$$

Details are discussed in SS. SS obtained almost identical results when the correction function consists of two sine modes:

$$
\delta h_{j}=a \sin \left[\alpha\left(h_{\max }-h_{j}^{*}\right)\right]+b \sin \left[2 \alpha\left(h_{\max }-h_{j}^{*}\right)\right]
$$

where $\alpha=\pi /\left(h_{\max }-h_{\min }\right), a$ and $b$ are constants to be determined. Hence, only (2.20) is used in this paper. 


\subsection{Conservation of Total Energy}

If we multiply (2.1) by $g h,(2.2)$ by $u$ and (2.3) by $v$, we obtain:

$$
\frac{\partial}{\partial t}\left[\frac{h\left(u^{2}+v^{2}\right)}{2}+\frac{g h^{2}}{2}\right]+\frac{\partial}{\partial x}\left\{\left[\frac{h\left(u^{2}+v^{2}\right)}{2}+g h^{2}\right] u\right\}=0 .
$$

If we define $h=h_{b}+h^{\prime}$, where $h_{b}$ is the basic height and $h^{\prime}$ is the perturbation, and integrate (2.30) over the entire domain between $n \Delta t$ and $(n+1) \Delta t$, we obtain:

$$
\begin{aligned}
& \left.\frac{\partial}{\partial t} \sum_{j m} \mid \frac{h_{j}\left(u_{j}^{2}+v_{j}^{2}\right)}{2}+\frac{g\left(h_{b}+h_{j}^{\prime}\right)^{2}}{2}\right] \delta x_{j} \\
& =\left\{\left[\frac{h_{1}\left(u_{1}^{2}+v_{1}^{2}\right)}{2}+g\left(h_{b}+h_{1}^{\prime}\right)^{2}\right] u_{1}\right\}^{n+1 / 2} \\
& -\left\{\left[\frac{h_{j m}\left(u_{j m}^{2}+v_{j m}^{2}\right)}{2}+g\left(h_{b}+h_{j m}^{\prime}\right)^{2}\right] u_{j m}\right\}^{n+1 / 2} .
\end{aligned}
$$

For a closed domain, or domain with a periodic boundary or far away boundaries, we obtain:

$$
\frac{\partial}{\partial t} \sum_{j m}\left\lfloor\frac{h_{j}\left(u_{j}^{2}+v_{j}^{2}\right)}{2}+\frac{g\left(h_{b}+h_{j}^{\prime}\right)^{2}}{2}\right\rfloor \delta x_{j}=0
$$

or

$$
\frac{\partial}{\partial t} \sum_{j m}\left\lfloor\frac{h_{j}\left(u_{j}^{2}+v_{j}^{2}\right)}{2}+\frac{g\left(h_{j}^{\prime}\right)^{2}}{2}\right\rfloor \delta x_{j}=0
$$

because $\sum_{i m} h_{j}^{\prime} \delta x_{j}$ is a constant according to the conservation of mass. Let us define the total Kinetic Energy, $K=\sum_{j m}\left\lfloor\frac{h_{j}\left(u_{j}^{2}+v_{j}^{2}\right)}{2}\right\rfloor \delta x_{j}$; and the total potential energy, $P=\sum_{j m}\left\lfloor\frac{g\left(h_{j}^{\prime}\right)^{2}}{2}\right\rfloor \delta x_{j}$. The total energy at the $n^{\text {th }}$ time step, $E^{n}=K^{n}+P^{n}$, should be conserved and equal to its initial value $E^{0}$. 
After solving $\hat{u}_{j}, \hat{v}_{j}$ from (2.16) - (2.17) and $h_{j}^{n+1}$ from (2.27), we will apply the variation formulation so that the total energy can be conserved with a minimum modification to those obtained by (2.16) and (2.17). If we define:

$$
\hat{\kappa}_{j}=\frac{h_{j}^{n+1}\left(\hat{u}_{j}^{2}+\hat{v}_{j}^{2}\right)}{2} \delta x_{j}
$$

the perturbation of $\delta \hat{\kappa}_{j}$ for a close or periodic system should satisfy the following constraint:

$$
\sum_{j m} \delta \hat{\kappa}_{j}=E^{0}-P^{n+1}-\sum_{j m} \hat{\kappa}_{j}
$$

Here $\delta \hat{\kappa}_{j}$ is similar to $\delta h_{j}$ in (2.20). We can follow the variation formulation $(2.20-2.22)$ to calculate $\delta \hat{\kappa}_{j}$ and the kinetic energy:

$$
k_{j}^{n+1}=\hat{\kappa}_{j}+\delta \hat{\kappa}_{j}=\frac{1}{2} h_{j}^{n+1}\left[\left(u_{j}^{n+1}\right)^{2}+\left(v_{j}^{n+1}\right)^{2}\right\rfloor \delta x_{j} .
$$

Then, we assume that the change of kinetic energy does not affect the wind direction, that is:

$$
u_{j}^{n+1}=\hat{u}_{j}\left(1+\delta_{j}\right)
$$

and

$$
v_{j}^{n+1}=\hat{v}_{j}\left(1+\delta_{j}\right)
$$

From (3.34) - (2.35), we can solve $\delta_{j}$ and consequently $\left(u_{j}^{n+1}, v_{j}^{n+1}\right)$. The formulation of (2.35a, b) also applies to the irrotational fluid $(f=0$ and $v=0)$.

The variation technique discussed here can be easily applied to multi-dimensional problems, as shown in SS, which conserves the total mass in two-dimensional flow.

\section{NUMERICAL MODEL}

The model based on A-grids consists of $j m$ grids with a uniform space interval $\delta x$, which is integrated for $\mathrm{N}$-time steps. The basic height $h_{b}=200 \mathrm{~m}$. The initial perturbed height $h^{\prime}$ is given by:

$$
h_{j}^{\prime}=h_{a}^{\prime} \exp \left\{-\left[\left(x_{j}-x_{c}\right) /(A \lambda)\right]^{2}\right\},
$$


where $h_{a}^{\prime}$ is the amplitude of height perturbation, $x_{j}$ and $x_{c}$ are the location at $j^{\text {th }}$-grid and the central point, respectively; $\lambda=\sqrt{\left(g h_{b}\right)} / 1.0^{-4}=447.2 \mathrm{~km}$, is the radius of deformation if $f=10^{-4} \mathrm{~s}^{-1}$ in a rotational fluid and $\mathrm{g}=10 \mathrm{~m} \mathrm{~s}^{-2}$; where $A$ is a parameter to control the length scale $L x$ of the initial disturbance, $L x=A \lambda$. We also calculate the error of the total mass $(E m)$ and error of the total energy (Ee) at the $N^{\text {th }}$ time step (last time step) as follows:

$$
\begin{aligned}
& E m=\left(H^{N}-H^{0}\right) / H^{0}=\left(\sum_{i m} h_{j}^{N} \delta x_{j}-\sum_{i m} h_{j}^{o} \delta x_{j}\right) / \sum_{i m} h_{j}^{o} \delta x_{j}, \\
& E e=\left(K^{N}+P^{N}-E^{0}\right) / E^{0} .
\end{aligned}
$$

Table 1 provides the parameters used in this study. The model is integrated with double precision.

\section{NUMERICAL RESULTS IN AN IRROTATIONAL SYSTEM}

In addition to pure gravity waves (i.e., $f=0$ ), the results will show the numerical simulations of geostrophic adjustment from the initial shortwave $(\mathrm{A}<1)$ and longwave $(\mathrm{A}>1)$ disturbances of either velocity or mass field.

\subsection{Case 4A}

The system starts from rest ( $u=v=0$ everywhere) and an initial perturbation $h^{\prime}$ given by (3.1) with $h_{a}^{\prime}=0.1 \mathrm{~m}, h_{b}=200 \mathrm{~m}, A=0.2, \delta t=320 \mathrm{~s}, \delta x=3.125 \mathrm{~km}, j m=1601$, and $f=0$, as indicated by curve A in Fig. 1a and Case 4A-a in Table 1. As time increases, the perturbation propagates as surface gravity waves, as shown by $\mathrm{B}, \mathrm{C}$, and D at $t=1.07 \times 10^{4}, 2.13 \times 10^{4}$, and $3.2 \times 10^{4} \mathrm{~s}$, respectively. Because the perturbation is very small, the simulation is almost identical to the analytic solution. The waves propagate with a phase of $44.72 \mathrm{~m} \mathrm{~s}^{-1}(=\sqrt{g h})$. The maximum Courant number $C N$, which is define as $C N=(\max |u|+\sqrt{g h}) \delta t / \delta x,=4.58$ with $\max |u| \approx 0.012 \mathrm{~m} \mathrm{~s}^{-1}$. The total kinetic energy $K$ is identical to the total potential energy $P$, as shown in Fig. 1b. This is called the principle of equipartition of energy and is valid in conservative dynamical systems undergoing small oscillations that are unaffected by planetary rotation (Kundu 1990). The error in mass field is very small in our simulations, as shown in Table 1.

Without applying the variation principle, the error of mass, $E m=0.5696 \times 10^{-11}$ and error of the total energy, $E e=-.2791 \times 10^{-6}$ are very small for Case 4A-a shown in Table 1, because the scheme is very accurate for advection equations (Sun et al. 1996; Sun and Yeh 1997; Sun and Sun 2004; Shieh et al. 2006).

Case 4A-b has the same initial perturbation as Case 4A-a, except for a coarse resolution $\delta x=12.5 \mathrm{~km}$ and $j m=401$. The results of Case 4A-b are very close to Case 4A-a except for the Courant number $=1.145$, and slightly larger errors, $E m=-0.90 \times 10^{-11}$ and $E e=-0.15 \times 10^{-3}$, as shown in Table 1. 


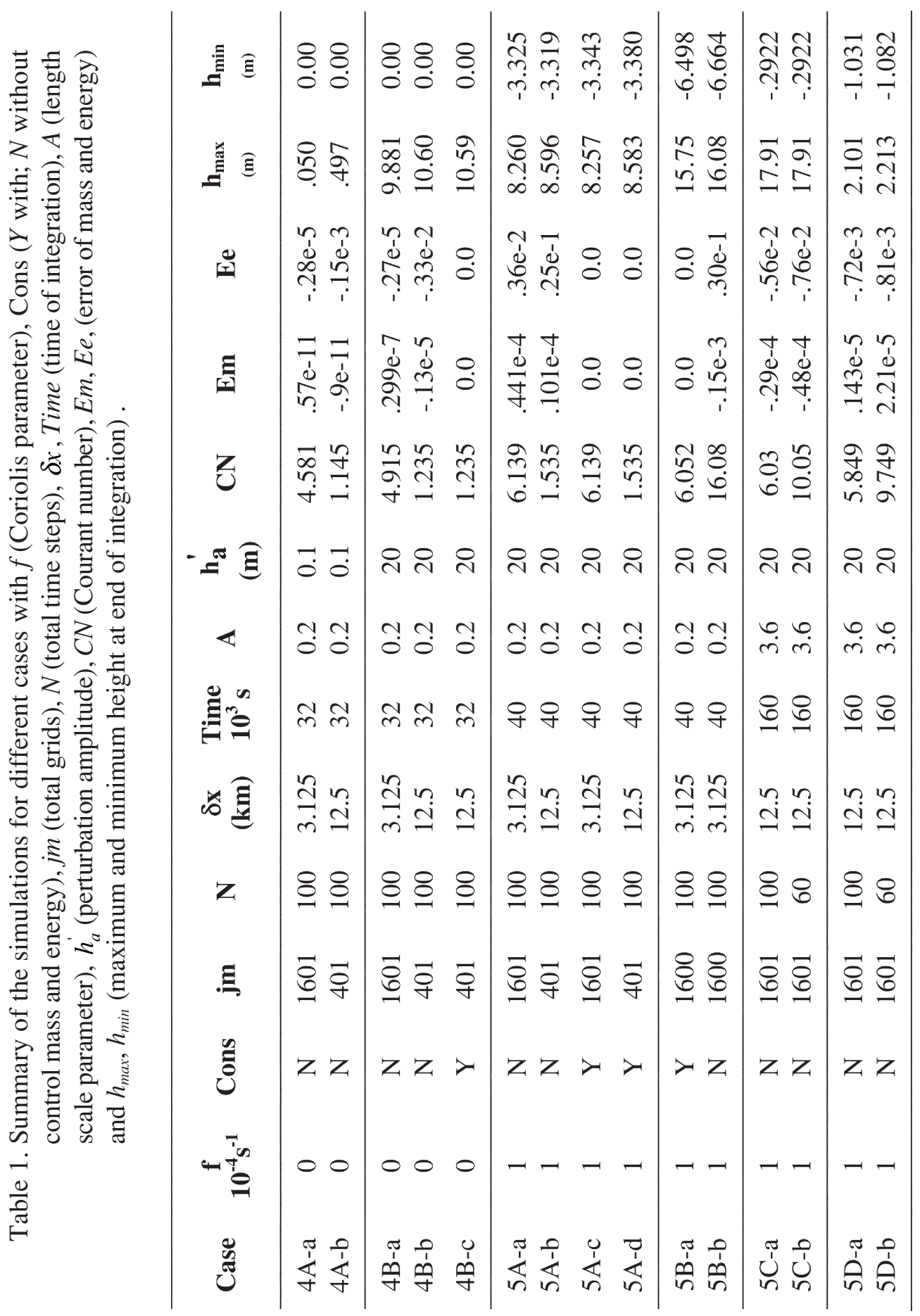


(a)

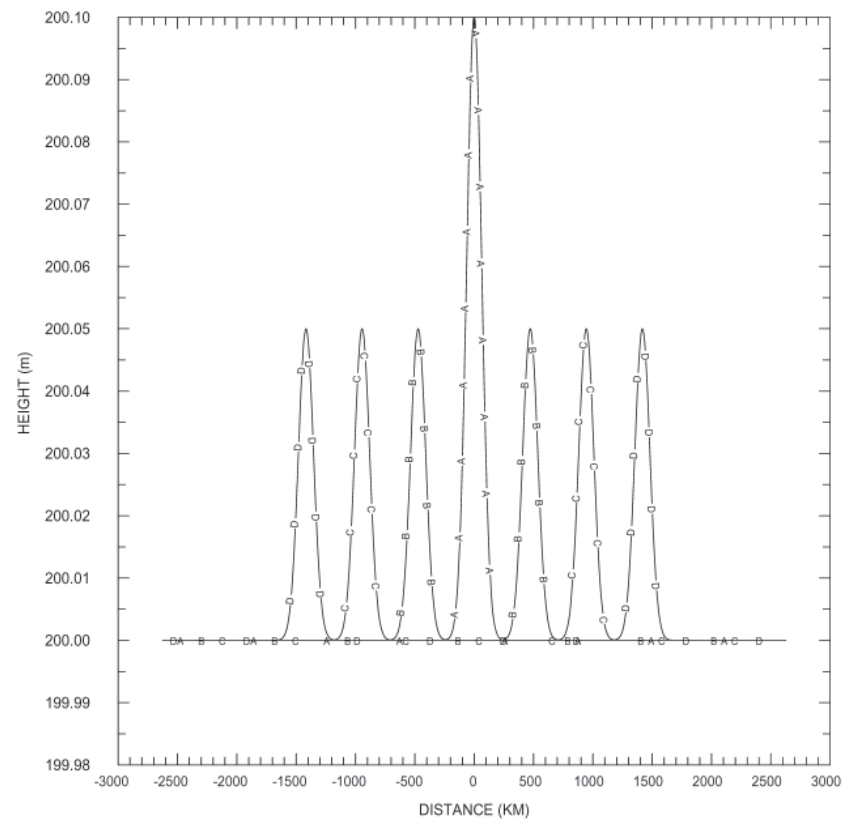

(b)

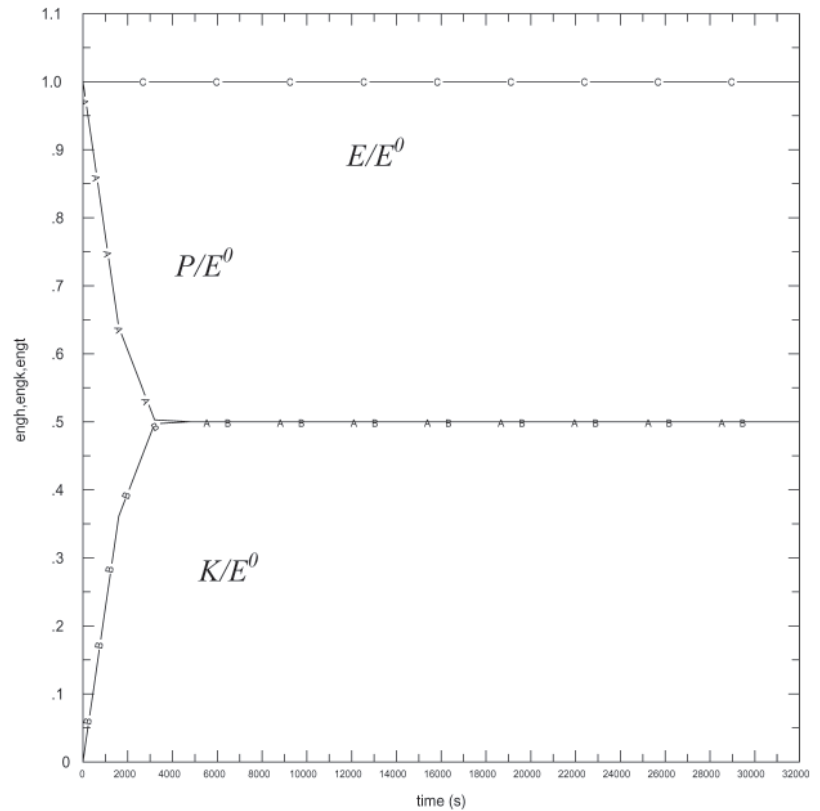

Fig. 1. Simulated: (a) $h$ at $t=0,1.07 \times 10^{4}, 2.13 \times 10^{4}$, and $3.2 \times 10^{4} \mathrm{~s}$, and (b) time-sequence of $E / E^{0}, K / E^{0}$, and $P / E^{0}$ for Case $4 \mathrm{~A}-\mathrm{a}$ with $h_{b}=200 \mathrm{~m}$, $h_{a}^{\prime}=0.1 \mathrm{~m}$, and $A=0.2$. 


\subsection{Case 4B}

Case 4B-a is the same as Case 4A-a except $h_{a}^{\prime}=20 \mathrm{~m}$, as shown in Table 1 and Fig. 2. Because the height is always symmetric and velocity is asymmetric with respect to the central point, $x_{c}$ in our simulations, hereafter, we will show the results for $x \geq x_{c}=0$ only. The Courant number $=4.915$ because of a stronger velocity $\left(\max |u| \approx 2.2 \mathrm{~m} \mathrm{~s}^{-1}\right)$ generated by a larger surface gradient. The phase speed is about $47 \mathrm{~m} \mathrm{~s}^{-1}$, which is carried by $u+c$ according to (2.9). The solutions depart from the linear solution as the tilt of both height field and velocity field increases with time. Similar results were obtained by Erbes (1993). Eventually, the wave collapses when the slope approaches infinity (i.e., $|\partial h / \partial x| \rightarrow \infty$ ), which is similar to collapse of the Burger equation discussed in Sun et al. (1996). The error in total mass is still very small, although it is larger than Cases of 4A. The error in total energy is comparable between 4A-a and $4 \mathrm{~B}-\mathrm{a}$. It is also noted that the ratio of $P / E^{0}$ in $4 \mathrm{~B}-\mathrm{a}$ is slightly less than 0.5 .

The solutions of Case 4B-b with coarse resolution $\delta x=12.5 \mathrm{~km}$ and $j m=401$ are also included in Table 1. The error in mass is still negligible, $E m=-.13 \times 10^{-5}$ but the error in the total energy, $E e=-.33 \times 10^{-2}$, which is much larger than the fine resolution simulation of Case 4B-a. On the other hand, Case 4B-c in table 1 shows that both mass and total energy can be conserved when the variation principle is applied. It is also noted that the difference in either height or velocity fields is very small for most points between Cases 4B-b and 4B-c.

(a)

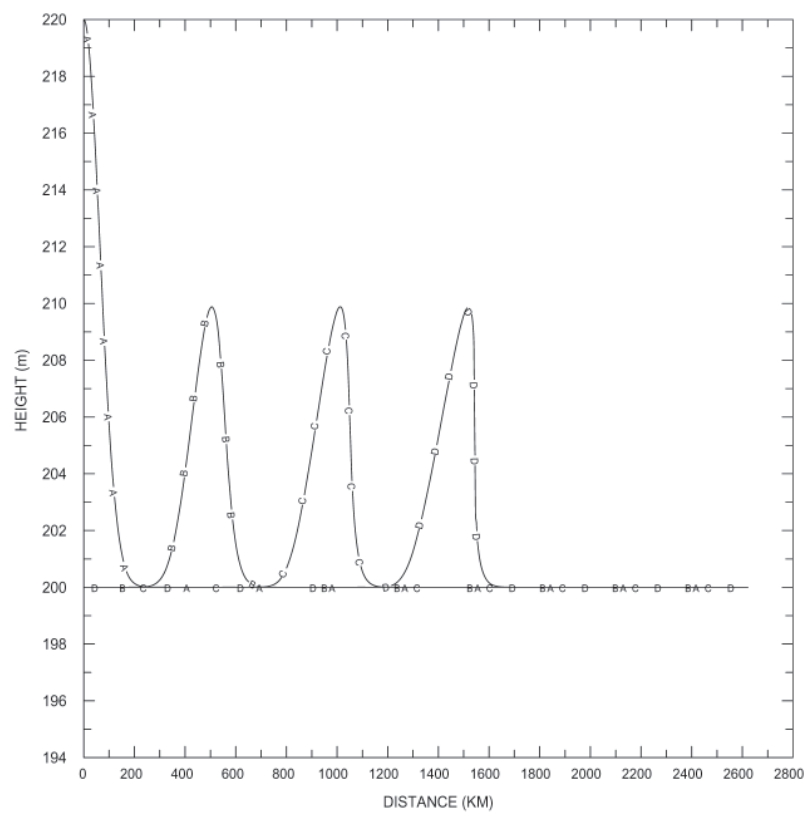

Fig. 2. Simulated: (a) $h$, (b) $u$ at $t=0,1.067 \times 10^{4}, 2.133 \times 10^{4}$ and $3.2 \times 10^{4} \mathrm{~s}$, and (c) time-sequence of $E / E^{0}, K / E^{0}$, and $P / E^{0}$ for Case $4 \mathrm{~B}$-a with $h_{b}=$ $200 \mathrm{~m}, h_{a}^{\prime}=20 \mathrm{~m}$, and $\mathrm{A}=0.2$. 
(b)

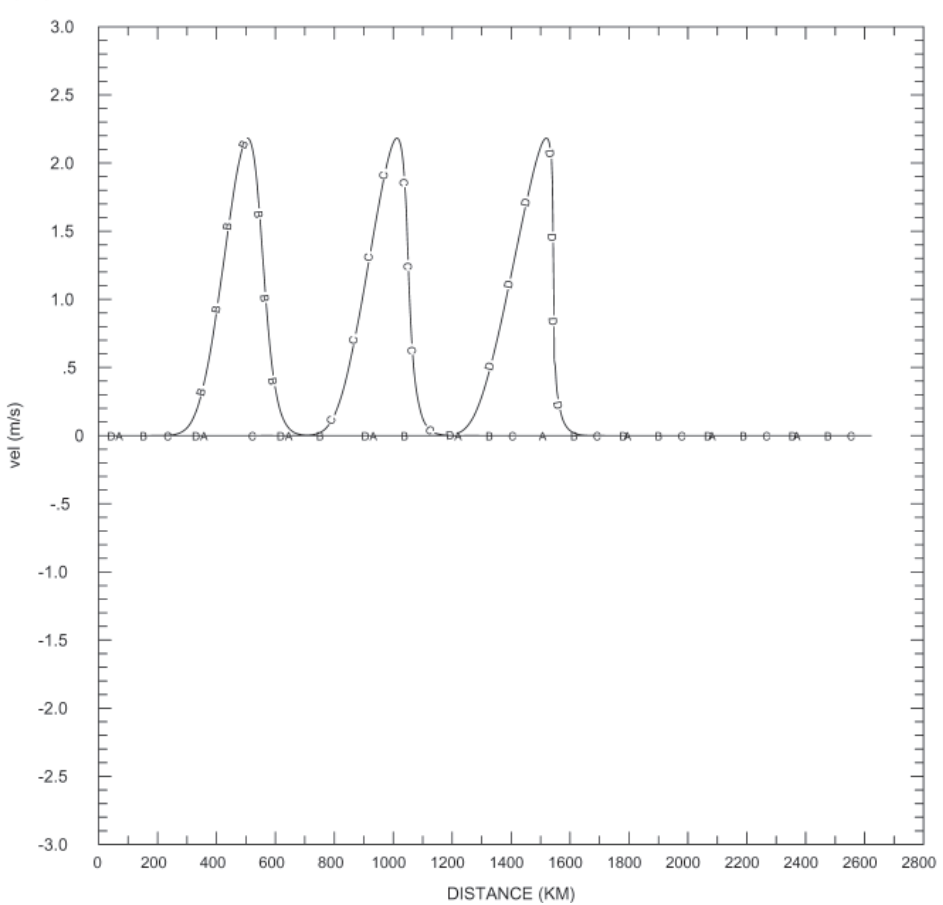

(c)

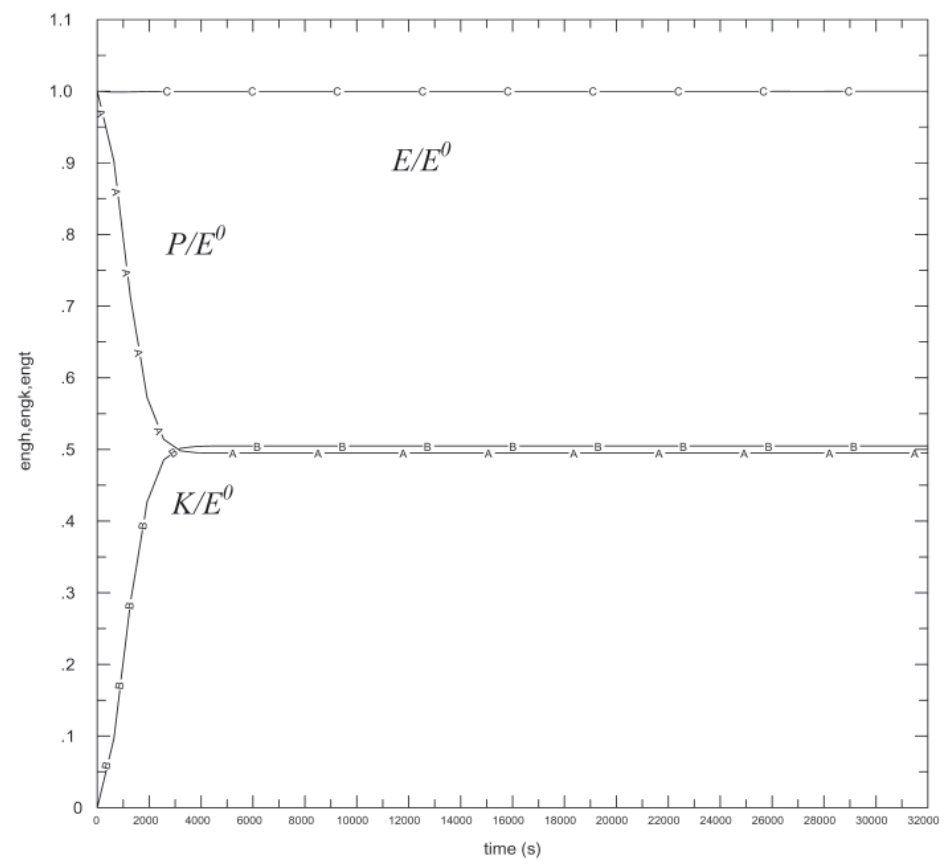

Fig. 2. (Continued) 


\section{RESULTS WITH THE CORIOLIS FORCE}

The propagation of waves becomes more complicated in a rotating system due to the existence of the Coriolis force (Rossby 1938; Gill 1982). The dispersion relation in linearized shallow water equations without mean flow becomes:

$$
\sigma^{2}=f^{2}+c^{2} k^{2}
$$

where $\sigma$ is frequency, $f=1.0^{-4} \mathrm{~s}^{-1}$ is the Coriolis parameter, and $k$ is the wave number. The phase speed of the dispersive wave is:

$$
c_{p}=\frac{\sigma}{\kappa}= \pm \sqrt{\frac{f^{2}}{k^{2}}+c^{2}} .
$$

Gravity (or buoyancy) effect is more important than the Coriolis force for short wave ( $c k>>f$ ). On the other hand, the Coriolis force becomes dominant for a long wave $(c k<<f)$.

\subsection{Case 5A}

The results for case 5A-a with $f=1.0^{-4} \mathrm{~s}^{-1}, h_{a}^{\prime}=20 \mathrm{~m}, A=0.2, \delta t=320 \mathrm{~s}, \delta x=3.125 \mathrm{~km}$, and $j m=1601$ but without conservations of mass and energy are shown in Table 1 and Fig. 3, where only half of the domain ( $x \geq x_{c}=0$ ) will be presented because $h$ is symmetric; while $u$ and $v$ are asymmetric with respect to $x_{c}$. Figures $3 \mathrm{a}-\mathrm{c}$ show the simulated $h, u$, and $v$ at $t=0$, $1.333 \times 10^{4}, 2.667 \times 10^{4}$, and $4 \times 10^{4} \mathrm{~s}$, which are indicated by A, B, C, and D, respectively. Figure $3 \mathrm{~d}$ reveals the time sequences of the total energy, potential energy, and kinetic energy. At the beginning, the unbalanced pressure gradient force in $x<\lambda$ generates the $\mathrm{x}$-component wind and the fast propagating gravity waves, the amplitude of the waves is smaller than that in Case 4B, because part of pressure gradient is counterbalanced by the y-component velocity, as we can see at $x=1 \times 10^{2} \mathrm{~km}$, the Coriolis force quickly turns a westerly wind to a northerly wind. Overshooting occurs in $x<\lambda$, thus, $h^{\prime}$ at $x=0$ decreases from 20 to $1.8 \mathrm{~m}$ within $13000 \mathrm{~s}$, then increases to $3.5 \mathrm{~m}$ at $t=40000 \mathrm{~s}$. The leading wave propagates away with a phase speed of $47.5 \mathrm{~m} \mathrm{~s}^{-1}$, which is similar to Case 4B but $0.5 \mathrm{~m} \mathrm{~s}^{-1}$ faster here due to the effect of rotation. The amplitude also gradually decreases with time.

The results reveal that for $x<100 \mathrm{~km}$, the $y$-component momentum reaches steady state within $20000 \mathrm{~s}$ while the height fields still changes with time because $L x$ is smaller than the radius of deformation $\lambda$ as shown in Figs. 3a - c.

The time sequence of energy (Fig. 3d) reveals that half of the potential energy quickly converts to the kinetic energy within $3000 \mathrm{~s}$. Then the energy conversion between potential energy and kinetic energy becomes much slower due to the inertia-gravity wave oscillation of the large scale waves shown in Figs. 3a - c. The amplitude of oscillation decreases slowly with time as the major portion of the system gradually approaches geostrophic balance.

When a coarse resolution, $\delta x=12.5 \mathrm{~km}$, is used, the simulated $h$ at $t=0,1.33 \times 10^{4}$, $2.66 \times 10^{4}$, and $4 \times 10^{4} \mathrm{~s}$ and the time evolution of energy are shown in Figs. $4 \mathrm{a}-\mathrm{b}$, which 
(a)

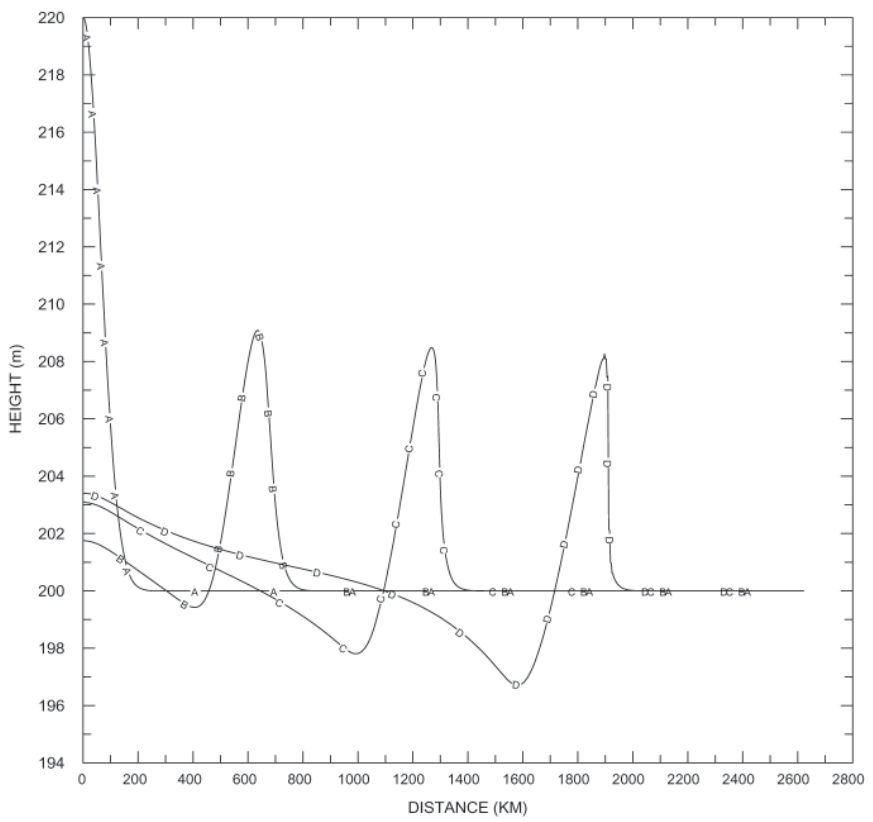

(b)

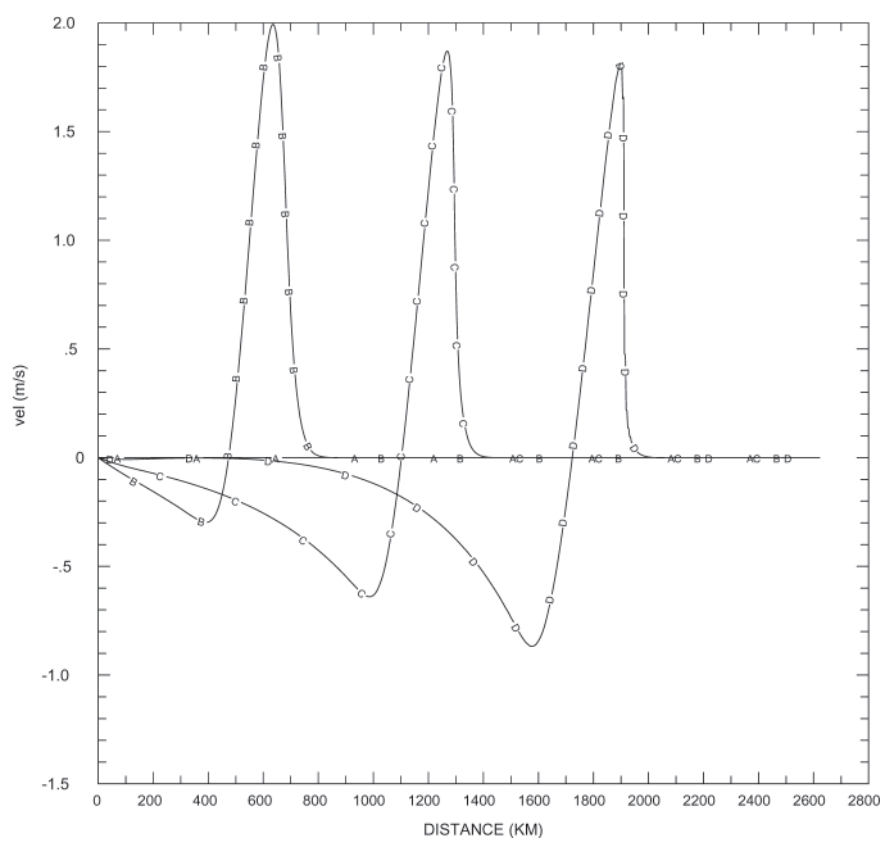

Fig. 3. Simulated: (a) $h$, (b) $u$, and (c) $v$ at $\mathrm{t}=0,1.333 \times 10^{4}, 2.666 \times 10^{4}$, and $4 \times 10^{4} \mathrm{~s}$; (d) time-sequence of $E / E^{0}, K / E^{0}$, and $P / E^{0}$ for Case $5 \mathrm{~A}$-a with $h_{b}=200 \mathrm{~m}, h_{a}^{\prime}=20 \mathrm{~m}, \mathrm{~A}=0.2$ and $\delta x=3.125 \mathrm{~km}$. 
(c)

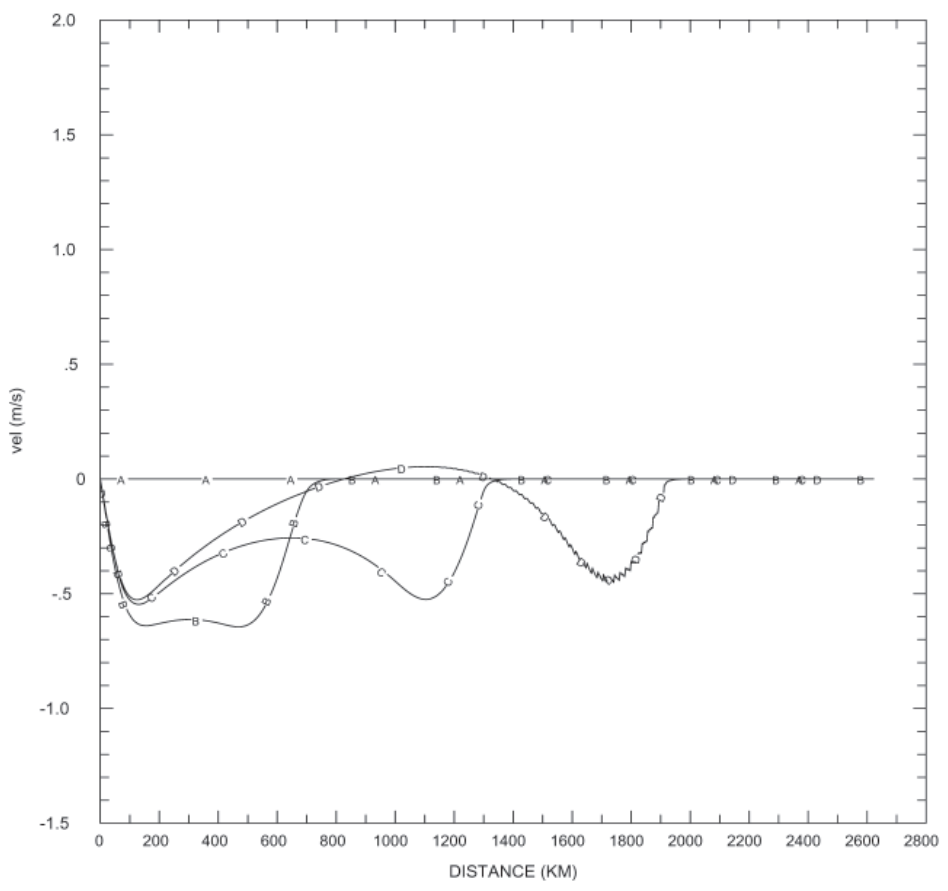

(d)

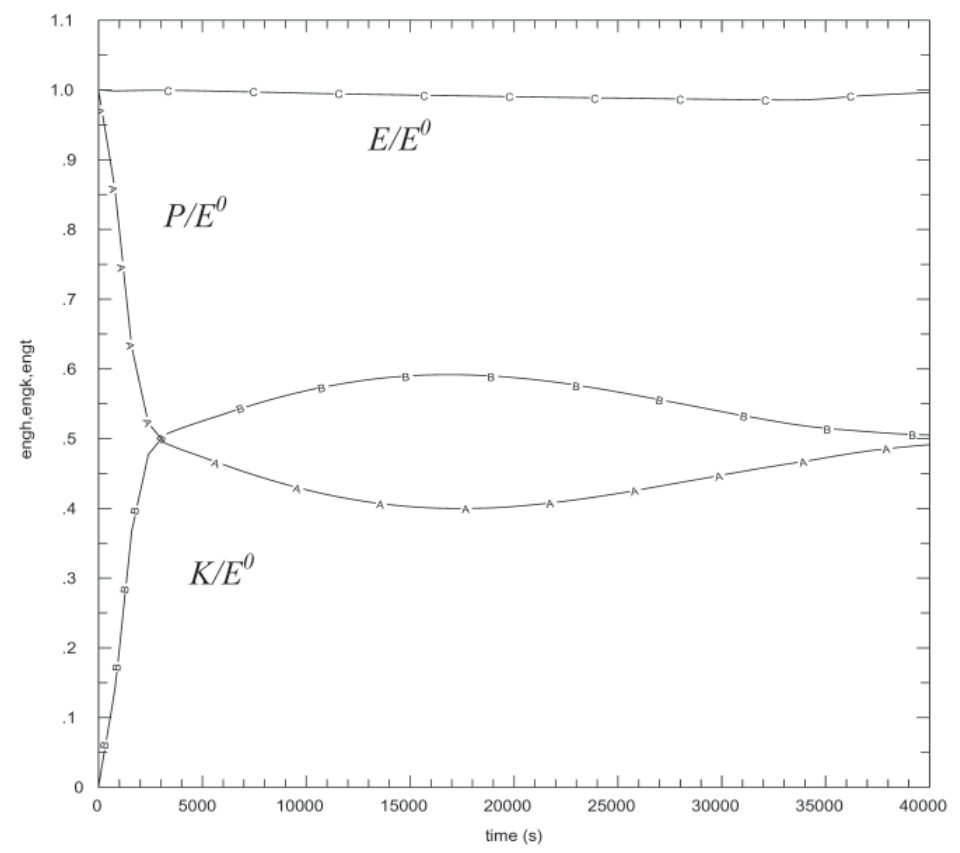

Fig. 3. (Continued) 
(a)

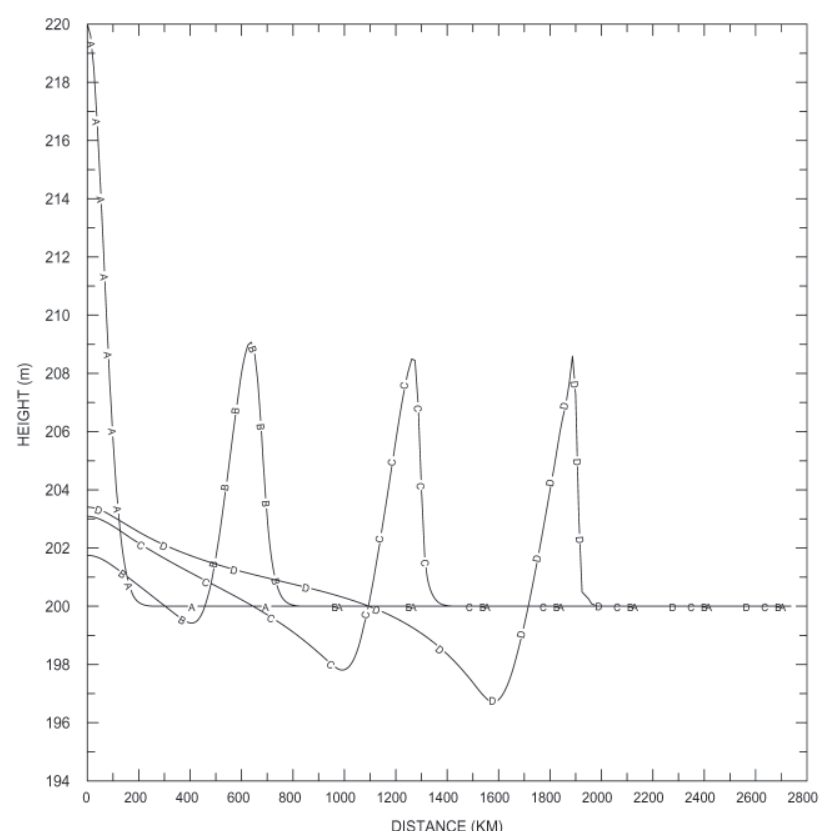

(b)

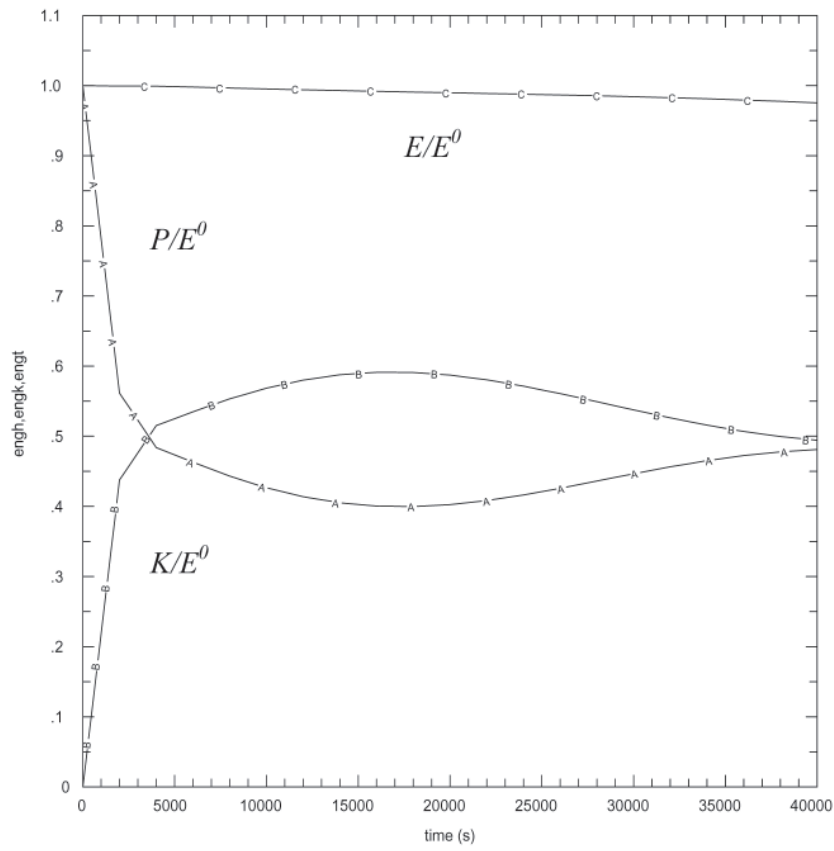

Fig. 4. Simulated: (a) $h$ at $t=0,1.333 \times 10^{4}, 2.666 \times 10^{4}$, and $4 \times 10^{4} \mathrm{~s}$, and (b) time-sequence of $E / E^{0}, K / E^{0}$, and $P / E^{0}$ for Case $5 \mathrm{~A}-\mathrm{b}$ with $h_{b}=200 \mathrm{~m}$, $h_{a}^{\prime}=20 \mathrm{~m}, \mathrm{~A}=0.2$, and $\delta x=12.5 \mathrm{~km}$. 
show a sharp peak in height field and $2.5 \%$ error in total energy at $t=40000 \mathrm{~s}$ as shown in Case $5 \mathrm{~A}-\mathrm{b}$ in Table 1. On the other hand, mass and total energy can be conserved when the variation principle is applied, as shown Case 5A-c and 5A-d in Table 1.

\subsection{Case 5B}

First, we introduce an initial perturbation $h^{\prime}$ as in Case 5A-a, then, a geostrophic wind balance is applied to obtain the y-component wind, which is shown as curve A in Fig. 5a for the right hand side of the domain $\left(x \geq x_{c}=0\right)$. After introducing $\mathrm{y}$-component wind, we reset to $h^{\prime}=0$ initially, as curve A in Fig. 5b. Hence, there is a y-component wind but no height perturbation initially. Furthermore, conservation of mass and total energy is also applied in Case 5B-a. The results are shown in Table 1 and Figs. 5a - d, in which we can see that the $y$-component wind does not change much with time within $x<200 \mathrm{~km}$. On the other hand, $h^{\prime}$ increases rapidly between $t=0$ and $t=1.33 \times 10^{4} \mathrm{~s}$ (Fig. 5b). The waves generated by an initial unbalanced force propagate away as inertia-gravity waves (Figs. $5 \mathrm{~b}-\mathrm{c}$ ). Because of a little adjustment in the momentum field, about $95 \%$ of the total energy remains as kinetic energy, and

(a)

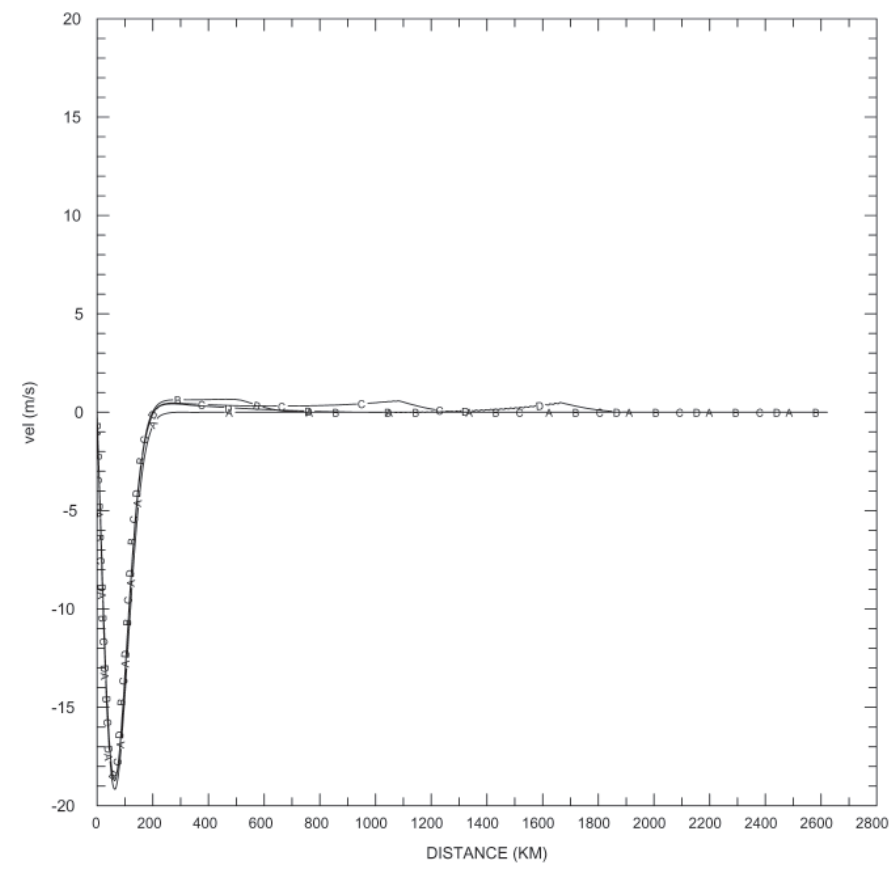

Fig. 5. Simulated: (a) $v$, (b) $h$, and (c) $u$ at $t=0,1.333 \times 10^{4}, 2.666 \times 10^{4}$, and $4 \times 10^{4} \mathrm{~s}$, and (d) time-sequence of $E / E^{0}, K / E^{0}$, and $P / E^{0}$ for Case $5 \mathrm{~B}$-a with $h_{b}=200 \mathrm{~m}, h_{a}^{\prime}=20 \mathrm{~m}, \mathrm{~A}=0.2$, and $\delta x=3.125 \mathrm{~km}$. (e) Same as Fig. $5 \mathrm{~d}$ except without controlling total mass or energy (Case 5B-b). 
(b)

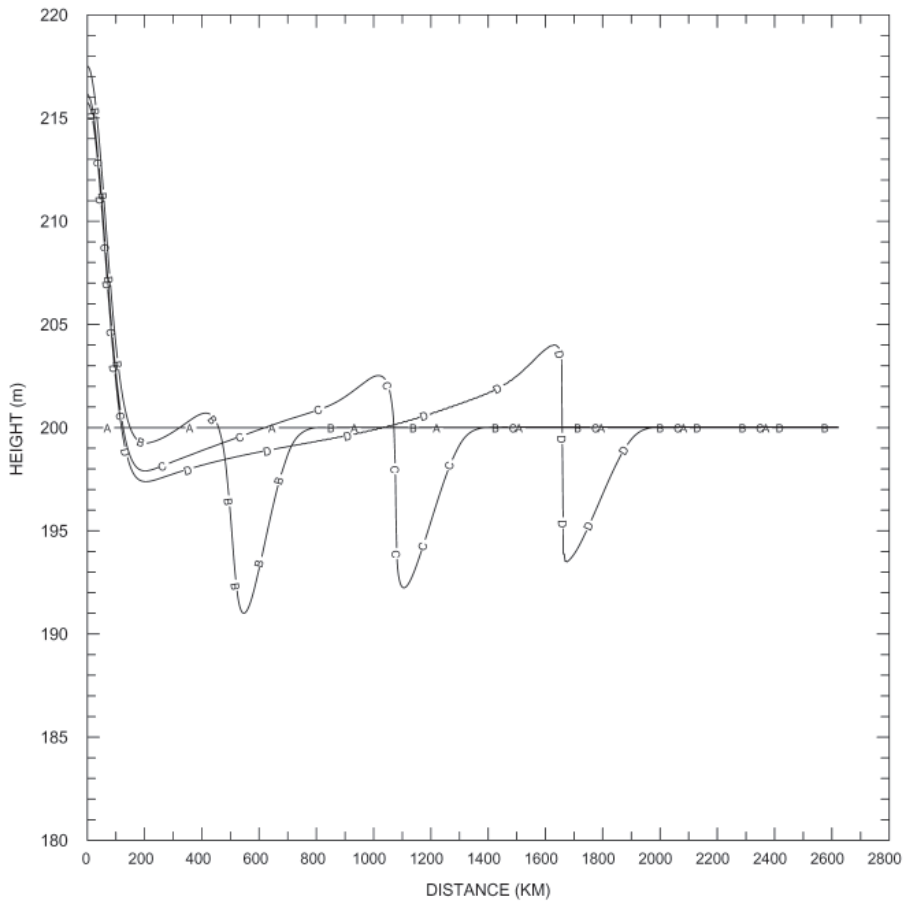

(c)

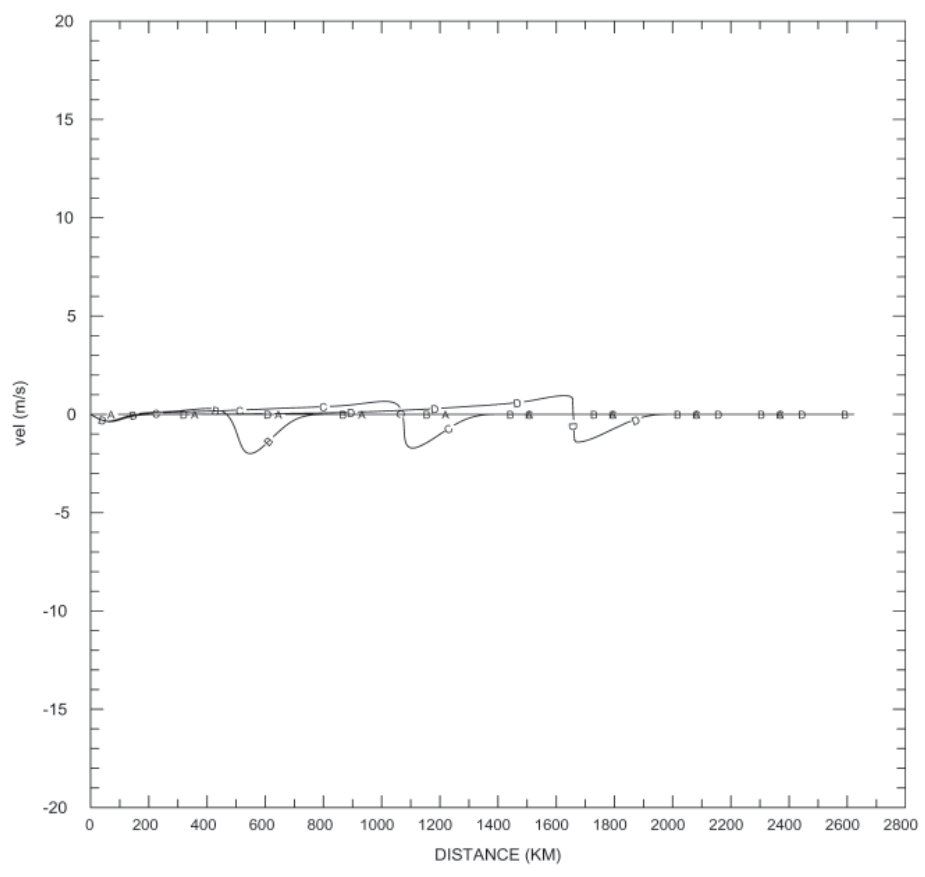

Fig. 5. (Continued) 
(d)

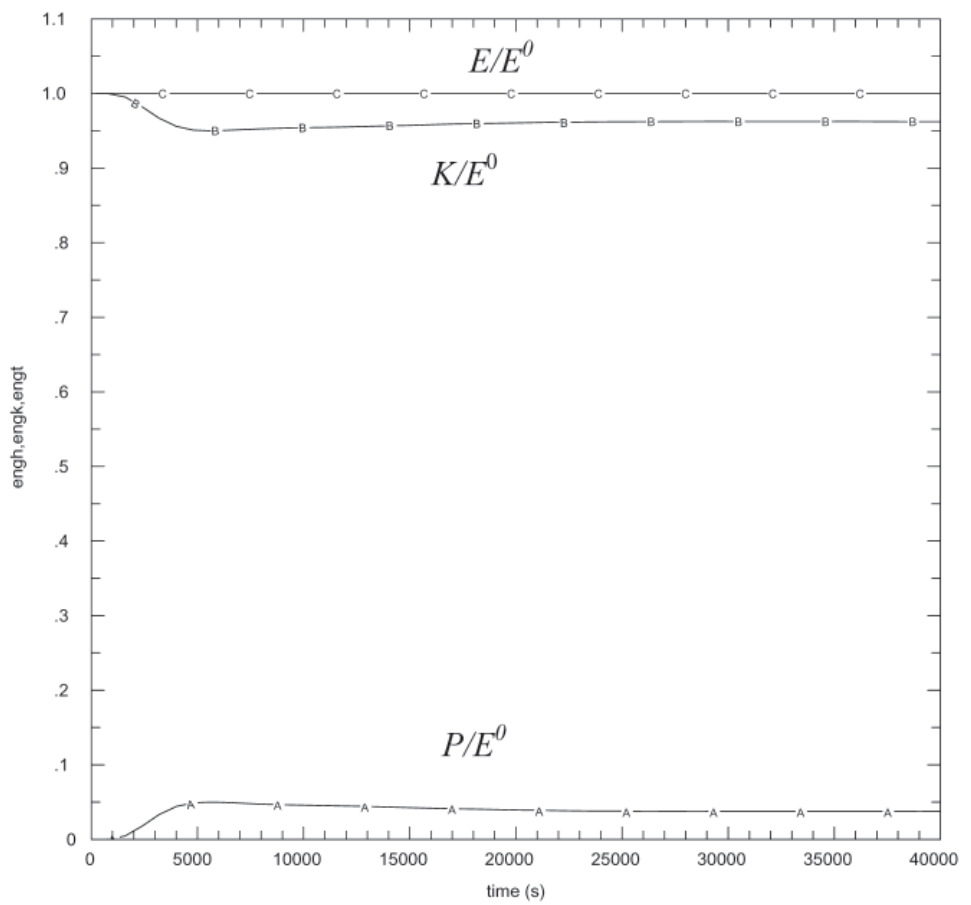

(e)

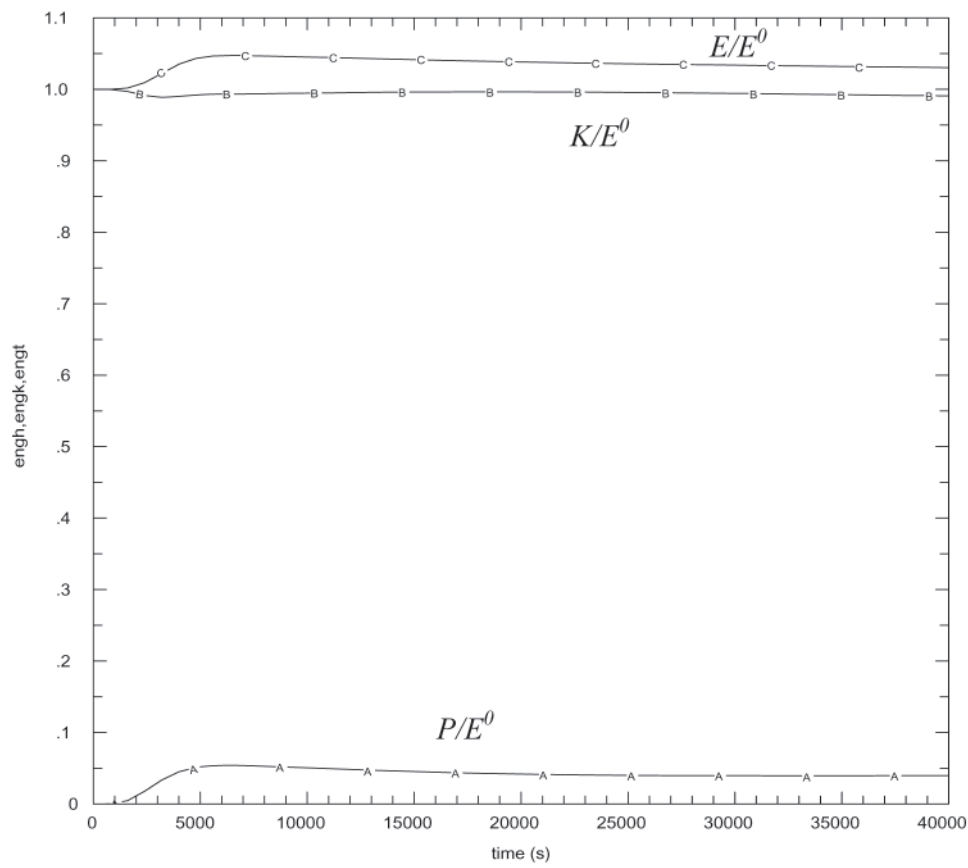

Fig. 5. (Continued) 
only $5 \%$ of the initial kinetic energy is converted to potential energy (Fig. 5d). Without applying the variation principle to control the total mass and energy, we obtain 3\% error in total energy, as shown in Fig. 5e and Case 5B-b in Table 1, although the difference in results between 5B-a and 5B-b are small in both velocity and height fields. The error in total mass is also small as shown in Table 1.

From Cases 5A-B, we can see that the mass field adjusts to the momentum field for an initial short wave perturbation (A $<<1$ ) according to geostrophic adjustment (Gill 1982).

\subsection{Case 5C}

The initial condition is the same as Case 5A, except $A=3.6$ and $\delta x=12.5 \mathrm{~km}$ and $\delta t=$ $1600 \mathrm{~s}(C N=6.02)$. The simulated heights for Case $5 \mathrm{C}$-a (without controlling for mass or energy) at $t=0,1.333 \times 10^{4}, 2.667 \times 10^{4}$, and $4 \times 10^{4} \mathrm{~s}$ are shown in Fig. $6 \mathrm{a}$, and the time sequence of velocity vector $(u, v)$ at $x=500,1300$, and $3825 \mathrm{~km}$ in Fig. $6 \mathrm{~b}$. Results show that the velocity adjusts to the mass field while around $90 \%$ of energy retains as potential energy,

(a)

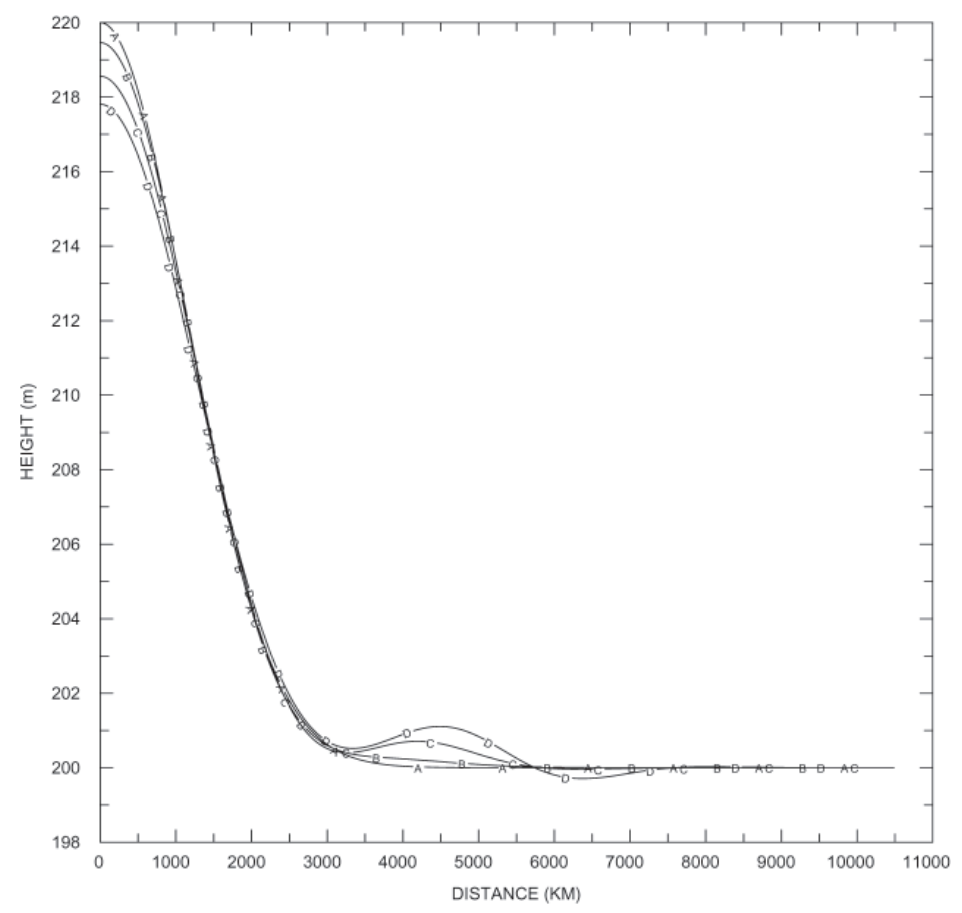

Fig. 6. Simulated: (a) $h$ at $t=0,5.33 \times 10^{4}, 1.07 \times 10^{5}$, and $1.6 \times 10^{5} \mathrm{~s}$, (b) timesequence of velocity $(u, v)$ at $500(\mathrm{X} 1), 1300(\mathrm{X} 2)$, and $3825(\mathrm{X} 3) \mathrm{km}$ and (c) time-sequence of energy $E / E^{0}, K / E^{0}$, and $P / E^{0}$ for Case $5 \mathrm{C}$-a with $h_{b}=200 \mathrm{~m}, h_{a}^{\prime}=20 \mathrm{~m}, \mathrm{~A}=3.2$ and $\delta x=12.5 \mathrm{~km}$. 
(b)

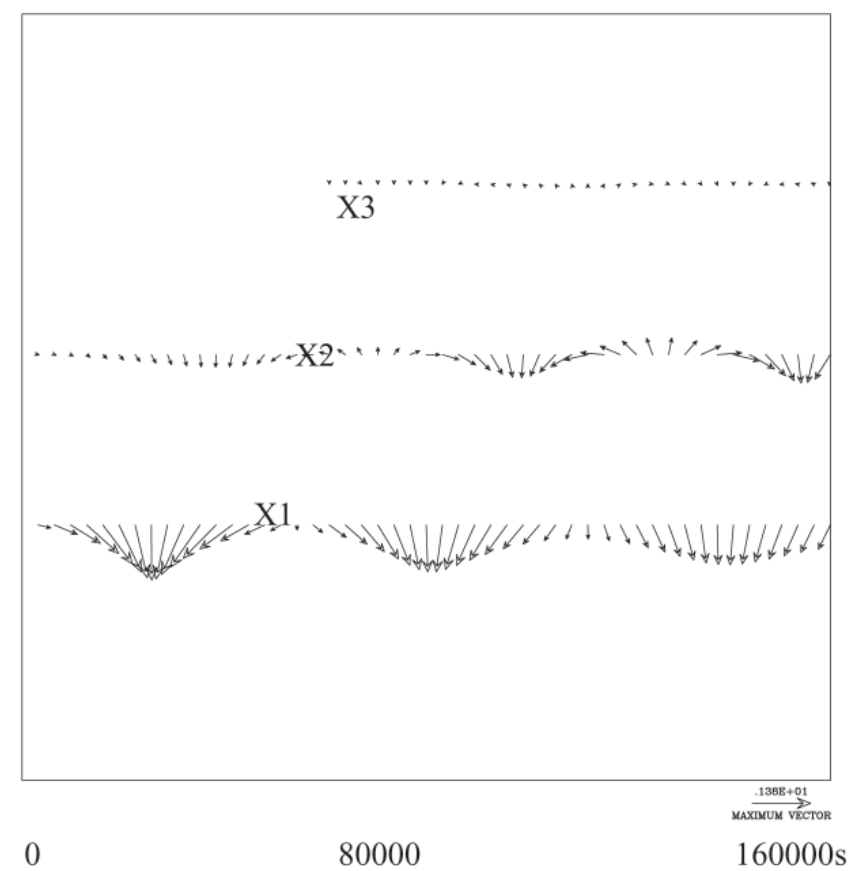

(c)

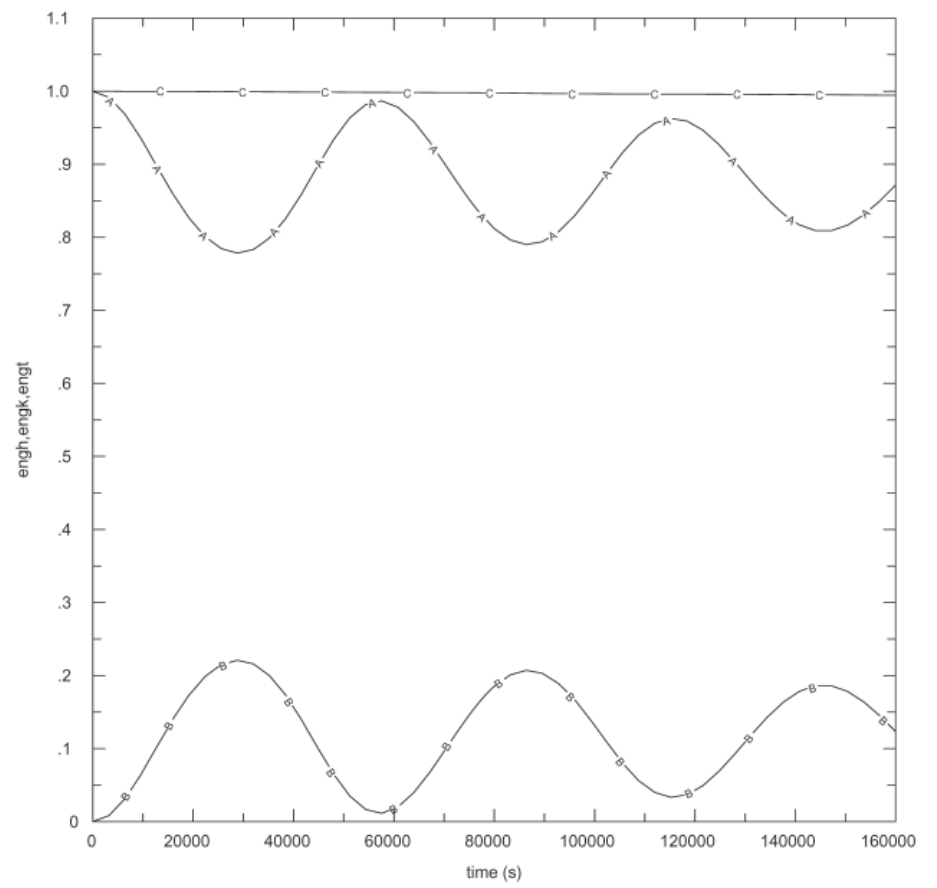

Fig. 6. (Continued) 
as shown in the time sequence of energy in Fig. 6c. Inertia-gravity oscillations exist in all fields with a period of about $57500 \mathrm{~s}$ with a small oscillation in mass (Fig. 6a) but a much larger oscillation in velocity field (Fig. 6b), although the waves are rather smooth compared with the disturbances generated by smaller scale perturbations in Cases 5A and 5B. The results remain about the same with $\delta t=2666.7 \mathrm{~s}$ and $C N=10.05$ (referred as Case 5C-b in Table 1). It is also noted that the errors in mass and total energy remain very small even without applying the variation principle.

\subsection{Case 5D}

The initial condition of Case 5D-a is the same as Case 5B-a, except $A=3.6$ here. Although about $75 \%$ of the energy remains as kinetic energy (Fig. 7c), the oscillation of both potential and kinetic energies is much larger than for Case $5 \mathrm{C}$-a. This indicates that it is slower to approach a geostrophic balance with an unbalanced large-scale momentum field as initial condition. Table 1 also shows that the errors for Case 5D-a are quite small even without controlling for mass or energy. The errors remain very small with a larger time interval $\delta t=2666.7 \mathrm{~s}$ as shown in Case 5D-b in Table 1.

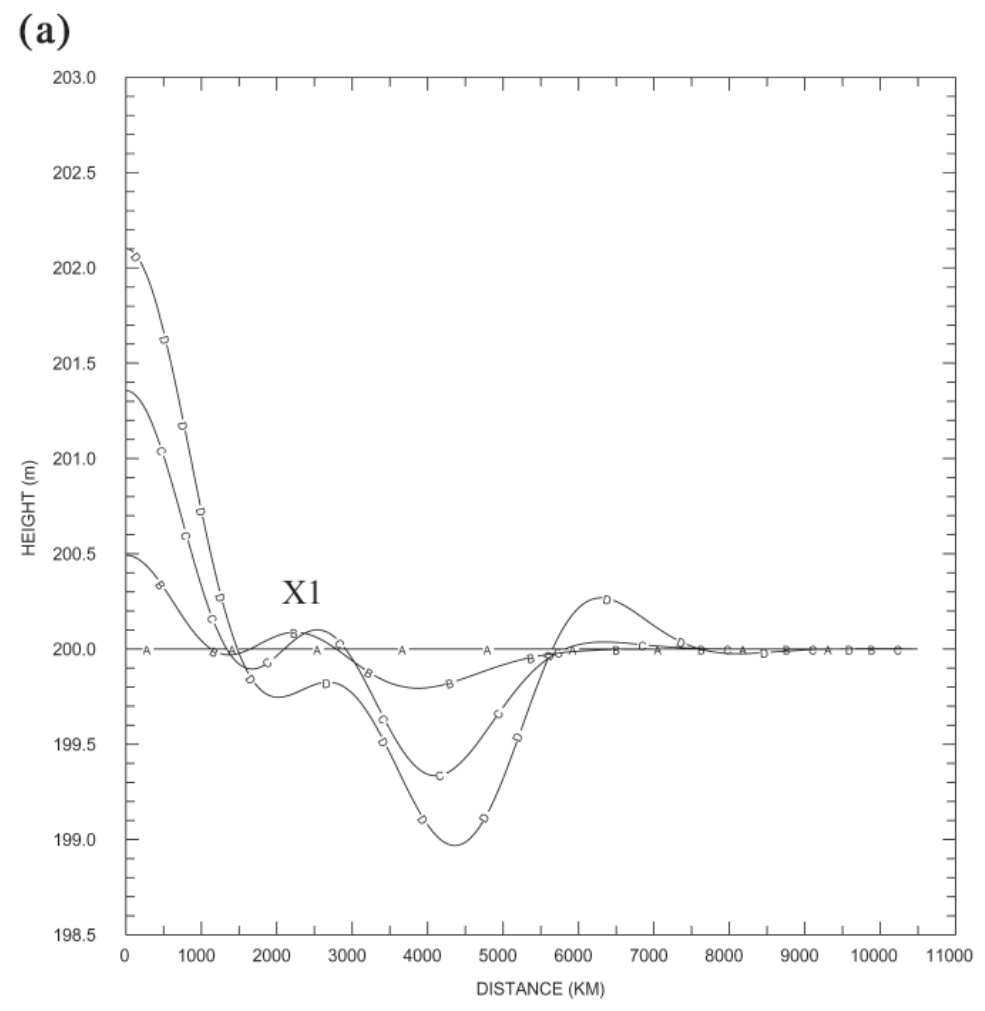

Fig. 7. same as Fig. 6 except for Case 5D-a. 
(b)

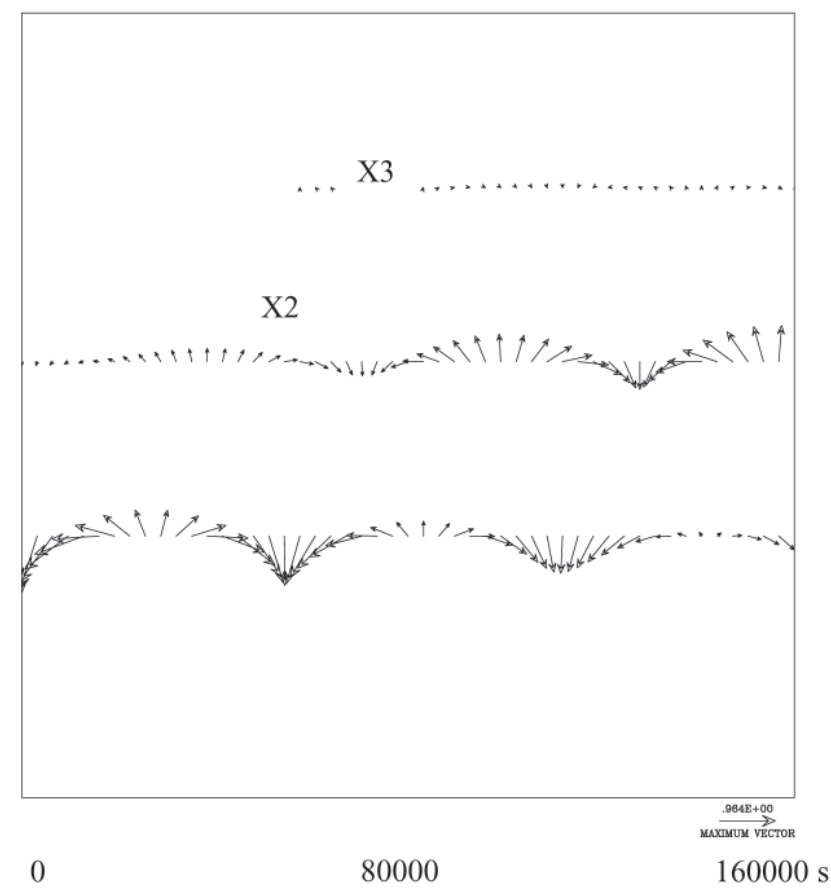

(c)

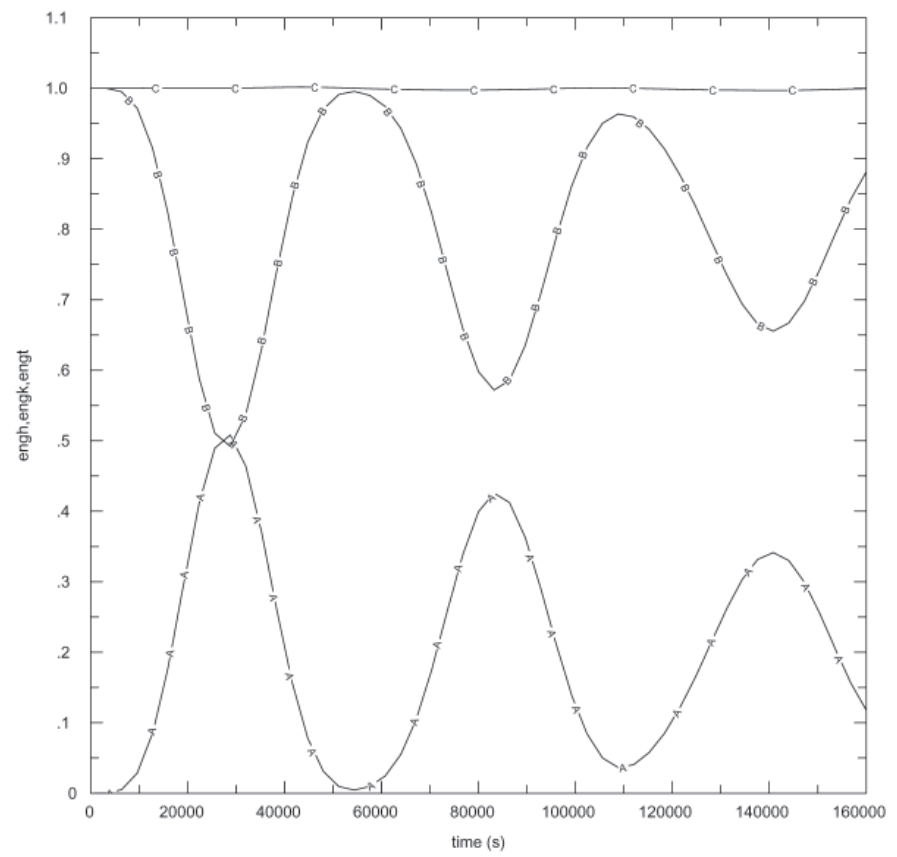

Fig. 7. (Continued) 
This paper reveals that we can apply the semi-Lagrangian scheme presented by Sun et al. (1996), Sun and Yeh (1997) to obtain accurate, positive-definite solutions from one-dimensional shallow water equations with or without the Coriolis force. According to SS, we can also add the variation formulations to guarantee the conservation of mass and total energy of the solutions.

Although in the 1-D case only left or right characteristic directions (i.e., $\pm x$ directions) exist, in the multi-dimensional cases there are an infinite number of characteristic directions that could be used for characteristic wave tracking, as discussed in Hirsch $(1988,1990)$ and Wang and Yeh (2005). Hence, the characteristic equations of the semi-Lagrangian system in 2-D or 3-D are quite complicated. However, the semi-Lagrangian method is much more accurate than the finite difference method when they are applied to simulate flow with a sharp gradient. After solving $u, v$, and $h$, we can easily apply the variation principle to achieve the conservation of mass and total energy in a 2-D or 3-D flow. Simulations for two-dimensional shallow water equations will be presented in future papers.

\section{SUMMARY}

A forward-in-time semi-Lagrangian scheme developed by Sun et al. (1996) and Sun and Yeh (1997) has been applied to one-dimensional shallow water equations in both rotational and irrotational systems. After obtaining the numerical results, we employ the variation principle (Sun and Sun 2004) to find the minimum corrections needed to adjust both height and total energy so that they are preserved. Because the minimum correction based on variation principle is applied, diffusion or dispersion introduced here is rather localized and is smaller than that applied by Bermejo and Conde (2002), as discussed in SS. The scheme produces accurate, positive-definite solutions while conserving both mass and total energy. The variation principles can significantly reduce the error in the total energy for coarse-resolution models; however, the improvement is less significant in the mass field because the original schemes produce quite accurate mass even without any adjustment. It is noted that most people are concerned with the conservation of mass, because total energy decays in a viscous flow. Whilst, an accurate calculation of the total energy should be important, it is more difficult than the mass field.

The simulations show that (non-dispersive) gravity waves are generated by an initial perturbation in an irrotational system. On the other hand, the flow in a rotational system goes through geostrophic adjustment; the waves generated by unbalanced forcing propagate away as inertia-gravity waves. Currently we are working on two-dimensional flows to study the interactions of vortices over topography and the problems discussed by Wang and Yeh (2005) based on the characteristic-based semi-Lagrangian method presented here. The characteristic equations and semi-Lagrangian scheme for multi-dimensional flows are quite complicated and will be presented in future papers.

Acknowledgement The author would like to thank Professor P. Smith and Mr. B. MacCall at Purdue University for proofreading, and the reviewers for valuable comments. Part of this work was supported by ARL and NSF under Grant Number 0408345-ATM of the USA, NSC 
under Grant Number 93-2745-P492 of Taiwan and National Center for High Performance Computing.

\section{REFERENCES}

Arakawa, A., and V. R. Lamb, 1981: A potential enstrophy and energy conserving scheme for the shallow water equations. Mon. Weather Rev., 109, 18-36.

Bates, J. R., 1984: An efficient semi-Lagrangian and alternating-direction implicit method for integrating the shallow-water equations. Mon. Weather Rev., 112, 2033-2047.

Bermejo, R., and A. Staniforth, 1992: The conversion of semi-Lagrangian advection schemes to quasi-monotone schemes. Mon. Weather Rev., 120, 2622-2632.

Bermejo, R., and J. Conde, 2002: A conservative quasi-monotone semi-Lagrangian scheme. Mon. Weather Rev., 130, 430.

Carpenter, Jr. R. L., K. K. Droegemeier, R. R. Woodward, and C. E. Hane, 1990: Application of the piecewise parabolic method (PPM) to meteorological modeling. Mon. Weather Rev., 118, 586-612.

Erbes, G., 1993: A semi-Lagrangian method of characteristics for the shallow-water equation. Mon. Weather Rev., 121, 3443-3452.

Gill, A. E., 1982: Atmospheric-Ocean Dynamics. Academic Press, 662 pp.

Gravel, S., and A. Staniforth, 1994: A mass conservative semi-Lagrangian scheme for the shallow wave equations. Mon. Weather Rev., 122, 243-248.

Hirsch, C., 1988: Numerical Computation of Internal and External Flows. Volume 1: Fundamentals of Numerical Discretization, John Wiley \& Sons, 515 pp.

Hirsch, C., 1990: Numerical Computation of Internal and External Flows. Volume 2: Computational Methods for Inviscid and Viscous Flows, John Wiley \& Sons, 691 pp.

Hsu, W. R., and W. Y. Sun, 2001: A time-split, forward-backward numerical model for solving a nonhydrostatic and compressible system of equations. Tellus, 53A, 279-299.

Huang, C. Y., 1994: Semi-Lagrangian advection schemes and Eulerian WKL algorithms. Mon. Weather Rev., 122, 1647-1658.

Kuo, H. C., and R. T. Williams, 1990: Semi-Lagrangian solutions to the inviscid Burgers equation. Mon. Weather Rev., 118, 1278-1288.

Lin, S. J., and R. B. Rood, 1996: Multimensional flux-form semi-Lagrangian transport schemes. Mon. Weather Rev., 124, 2046-2069.

Nair, R., and B. Machenhauer, 2002: The mass-conservative cell-integrated semi-Lagrangian advection scheme on the sphere. Mon. Weather Rev., 130, 649-667.

Priestley, A., 1993: A quasi-conservative version of the semi-Lagrangian advection scheme. Mon. Weather Rev., 121, 621-629.

Purser, R. J., and L. M. Leslie, 1991: An efficient interpolation procedure for high-order three-dimensional semi-Lagrangian models. Mon. Weather Rev., 119, 2492-2498.

Purser, R. J., and L. M. Leslie, 1994: An efficient semi-Lagrangian scheme using third-order 
semi-implicit time integration and forward trajectories. Mon. Weather Rev., 122, 745756.

Rancic, M., 1992: Semi-Lagrangian piecewise biparabloic scheme for two-dimensional horizontal advection of a passive scalar. Mon. Weather Rev., 120, 1394-1406.

Rossby, C. G., 1938: On the mutual adjustment of pressure and velocity distribution incertain simple current systems, II. J. Marine Res., 239-263.

Shieh, M. N., W. R. Hsu, and W. Y. Sun 2006: Applications of a monotone semi-Lagrangian advection scheme on a 3D nonhydrostatic atmospheric model. The $13^{\text {th }}$ National computational Fluid Dynamics Conference. Taipei County, Taiwan, August 2006, CFD130107.

Staniforth, A., and J. Côté, 1991: Semi-Lagrangian integration schemes for atmospheric models - A review. Mon. Weather Rev., 119, 2206-2223.

Stoker, J., 1957: Water waves: The mathematics theory with Applications. Interscience, $567 \mathrm{pp}$.

Sun, W. Y., K. S. Yeh, and R. Y. Sun, 1996: A simple semi-Lagrangian scheme for advection equations. Quart. J. R. Meteorol. Soc., 122, 1211-1226.

Sun, W. Y., and K. S. Yeh, 1997: A general semi-Lagrangian advection scheme employing forward trajectories. J. R. Meteorol. Soc., 123, 2463-2476.

Sun, W. Y., and M. T. Sun, 2004: Mass correction applied to semi-Lagrangian advection scheme. Mon. Weather Rev., 132, 975-984.

Sun, W. Y., and W. R. Hsu, 2005: Effect of surface friction on downslope wind and mountain waves. Terr. Atmos. Ocean. Sci., 16, 393-418.

Thuburn, J., and A. Staniforth, 2004: Conservation and linear Rossby-mode dispersion on the spherical C grid. Mon. Weather Rev., 132, 641-653.

Wang, H., and G. T. Yeh, 2005; A characteristic-based semi-Lagrangian method for hyperbolic systems of conservation laws. Chinese J. Atmos. Sci., 29, 21-42.

Wiin-Nielsen, A., 1959: On the application of trajectory methods in numerical forecasting. Tellus, 11, 180-196.

Yih, C. S., 1979: Fluid Mechanics. West River Press, 622 pp.

Sun, W. Y., 2007: Conservative semi-Lagrangian scheme applied to one-dimensional shallow water equations. Terr. Atmos. Ocean. Sci., 18, 777-803, doi: 10.3319/TAO.2007. 18.4.777(A). 